\title{
Far-infrared photoconductivity in self-organized InAs quantum dots
}

\author{
J. Phillips, K. Kamath, and P. Bhattacharya ${ }^{a)}$ \\ Department of Electrical Engineering and Computer Science, Solid State Electronics Laboratory, \\ The University of Michigan, Ann Arbor, Michigan 48109-2122
}

(Received 6 January 1998; accepted for publication 23 February 1998)

\begin{abstract}
We report far-infrared photoconductivity in self-organized InAs/GaAs quantum dots grown by molecular beam epitaxy. Through use of a Fourier transform infrared spectrometer, a photoconductivity signal peaked at $17 \mu \mathrm{m}$ is observed from a $n-i-n$ detector structure with doped InAs quantum dots in the intrinsic region. Comparison of photoluminescence and band-to-band photocurrent absorption spectra suggests the far-infrared response is due to intersubband transitions in the quantum dots. (C) 1998 American Institute of Physics. [S0003-6951(98)04216-8]
\end{abstract}

The mid- and far infrared are useful for applications such as chemical spectroscopy, atmospheric communications, and image recognition. There is a great deal of interest in obtaining semiconductor detectors in this region. Semiconductor detectors in this wavelength range, such as $\mathrm{HgCdTe}$ and antimonides, have demonstrated much success, but require cooling and difficult process technologies. The use of bound-state transitions in quantum confined structures allows the use of more advanced process technologies, such as GaAs and InP, to achieve mid- and far-infrared detection. Quantum well infrared photodetectors ${ }^{1,2}$ have been successful in this application, but also require cooling and have a disadvantage in that they are not intrinsically sensitive to normal incidence photoexcitation. Quantum dots ideally have several advantages over quantum wells. Quantum dots are intrinsically sensitive to normal incidence photoexcitation, eliminating the need for gratings, reflectors, or engineered band mixing, as is required for quantum wells. Due to their discrete density of states, they should be useful for tunable narrow-band detection. Perhaps most importantly, due to reduced phonon scattering in quantum dots as compared to quantum wells, ${ }^{3,4}$ photoexcited carriers will be swept away as photocurrent before relaxing to the ground state, providing more efficient detection, and therefore, better quantum efficiency, and possibly allowing for increased temperature operation.

One highly successful method of achieving quantum dots, which has received much attention as of late, is through self-organized growth ${ }^{5-9}$ in the Stranski-Krastanow growth mode. The carrier capture times of self-organized quantum dot lasers ${ }^{10}$ have been recently measured ( $\left.\sim 40 \mathrm{ps}\right)$ and are comparatively longer than those measured for quantum well lasers $(\sim 5-10 \mathrm{ps})$. These times should further increase and prove advantageous for unipolar intersubband devices due to the absence of electron-hole scattering, which is predicted to be a dominant scattering mechanism in quantum dots. ${ }^{4}$ Farinfrared absorption has been observed in self-organized quantum dots. ${ }^{11-13}$ Detectors, however, also require the ability to extract excited carriers to contribute to a photocurrent signal. Midinfrared photoconductivity has been recently reported in self-organized quantum dots. ${ }^{14}$ By varying growth conditions and materials, self-organized quantum dots should

${ }^{a)}$ Electronic mail: pkb@eecs.umich.edu have the ability to provide wavelength tunable mid- and farinfrared detectors. In this letter, we report far-infrared photoconductivity in self-organized InAs quantum dots in the 10-20 $\mu \mathrm{m}$ range.

A detector structure was grown by molecular beam epitaxy on semi-insulating GaAs (100) substrates for farinfrared photoconductivity measurements. The grown structure is shown in Fig. 1 and consists of an intrinsic GaAs region with ten layers of self-organized InAs quantum dots, sandwiched between highly doped GaAs regions. Each InAs quantum dot layer consisted of a nominal InAs thickness of $7.5 \AA$ and was grown at $510^{\circ} \mathrm{C}$ at a growth rate of $0.3 \AA / \mathrm{s}$ with a background arsenic pressure of $1 \times 10^{5}$ T. A $10 \mathrm{~s}$ pause was used after the InAs growth to further promote quantum dot formation. The growth of InAs layers were monitored by reflection high-energy electron diffraction where the transition from two-dimensional to threedimensional island growth was observed after $\sim 1.8 \mathrm{ML}$ of InAs growth. Previous measurements on samples grown under similar conditions indicate a pyramidal dot shape with a typical base width of $17 \mathrm{~nm}$ and height of $7 \mathrm{~nm}$ and a dot density of $10^{10} \mathrm{~cm}^{-2}$ through transmission electron microscope and atomic force microscope images. The InAs quantum dot layers were uniformly and directly silicon doped, to provide carriers for absorption, to a level of $n=5$ $\times 10^{17} \mathrm{~cm}^{-3}$, corresponding to about 1 dopant atom/dot. The $\mathrm{GaAs}$ regions were grown at $630{ }^{\circ} \mathrm{C}$ with temperature ramps before and after quantum dot growth. Circular mesa structures of $400 \mu \mathrm{m}$ diam were patterned to contact top and

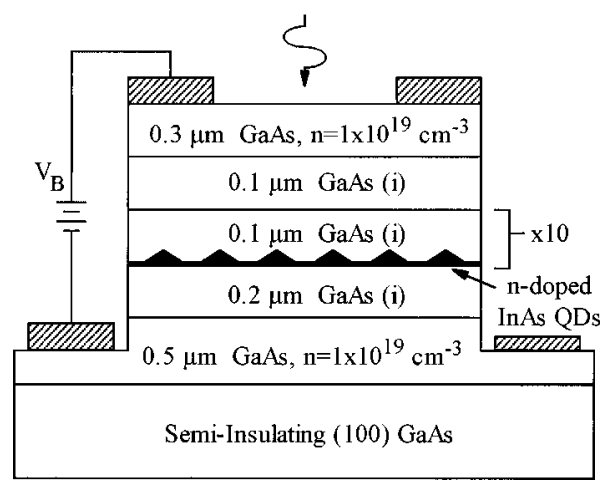

FIG. 1. Schematic of the growth and device structure with self-organized InAs quantum dots. 


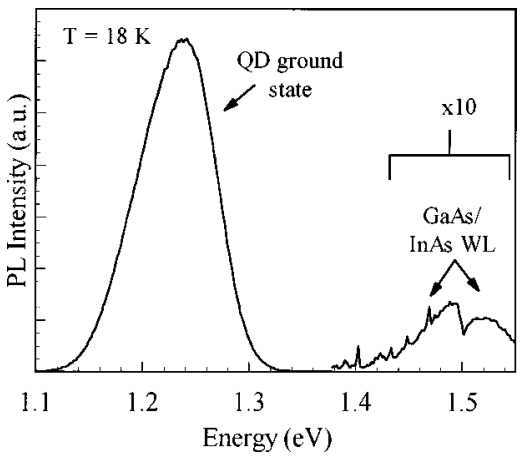

FIG. 2. Photoluminescence spectra ( $T=18 \mathrm{~K})$ of $n-i-n$ detector structure.

bottom $n^{+}$-GaAs regions. Ni/Ge/Au ring contacts were then defined and annealed for Ohmic contacts, and the devices were wire bonded for measurement. The device structure is shown schematically in Fig. 1.

Low-temperature $(T=18 \mathrm{~K})$ photoluminescence (PL) measurements were done using a $\mathrm{HeNe}(\lambda=632.8 \mathrm{~nm})$ laser, a $1 \mathrm{~m}$ monochrometer, a photomultiplier, and a lock-in amplification. The PL spectra of the heterostructure, shown in Fig. 2, shows a clear peak at $1.24 \mathrm{eV}$ originating from the quantum dots. Additional weaker luminescence peaks are observed at higher energies, which we attribute to GaAs luminescence with a possible contribution from the InAs wetting layer. The PL spectra does not indicate luminescence corresponding to excited-state transitions in the quantum dots.

Photocurrent spectra measurements were made to examine band-to-band transitions in the quantum dots. Measurements were made using light from a tungsten lamp dispersed by a $1.0 \mathrm{~m}$ monochrometer and lock-in amplification. The $n-i-n$ detector was biased by $(V=1.0 \mathrm{~V})$ and the photocurrent signal was measured through a low-noise current amplifier connected directly to the device. The photocurrent spectrum $(T=12 \mathrm{~K})$ is shown in Fig. 3 showing a strong absorption from GaAs in the intrinsic region and absorption from the quantum dots. Due to the discrete energy levels in the density of states of quantum dots, the band-to-band absorption in quantum dots is expected to show resonant absorption features. There are no clear peaks observed in the photocurrent spectra corresponding to band-to-band absorption in the quantum dots, but there is clearly a photocurrent

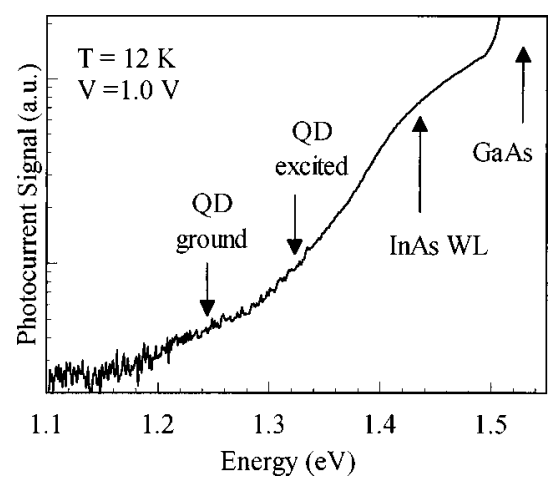

FIG. 3. Photocurrent spectra $(T=12 \mathrm{~K})$ of the $n-i-n$ detector structure with designations for band-to-band absorption in GaAs, InAs wetting layer, and InAs quantum dots, which are estimated from photoluminescence and far-infrared absorption measurements.

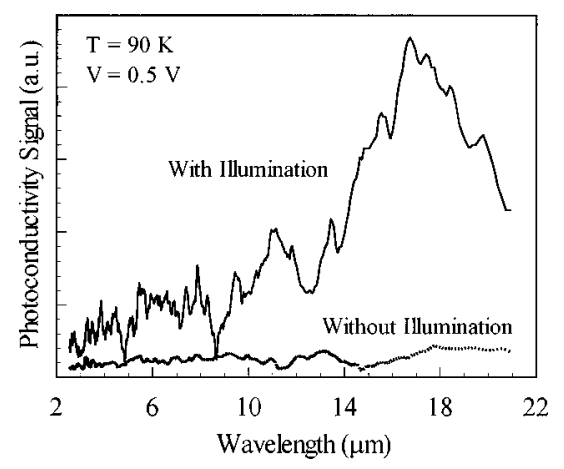

FIG. 4. Photoconductivity spectra $(T=90 \mathrm{~K})$ obtained using a FTIR spectrometer for normal incidence illumination and, for comparison, without illumination.

signal measured. The resonant absorption features may be absent due to the ensemble of dot sizes, which severely broadens the resonant absorption energies, and the inability for electron-hole pairs created in the quantum dots to escape the barrier energy to contribute to the photocurrent signal. An increased bias voltage was used to attempt to solve the latter problem, but resulted in saturation of the current amplifier. The varying change in slope of the photocurrent signal, however, suggests resonant absorption features near $1.43,1.32$, and $1.24 \mathrm{eV}$, which we believe are due to the InAs wetting layer and quantum dot excited and ground states, respectively. These assignments are supported by the following facts. The energy of the InAs wetting layer transition is almost identical to that measured. The peak energy of the PL transition in Fig. 2, due to the quantum dot ground state, is also at $1.24 \mathrm{eV}$. Finally, our finite impulse response data from $n$-doped InAs dots grown under similar conditions, which also show similar PL characteristics indicating similar dot size and distribution, revealed a transition at $18 \mu \mathrm{m} .{ }^{13}$ The corresponding energy difference is $70 \mathrm{meV}$, which nearly agrees with the difference $1.32-1.24 \mathrm{eV}$. We, therefore, expect a far-infrared photoconductive signal near or slightly larger than $16 \mu \mathrm{m}$ for the $n-i-n$ detector under study.

Far-infrared photoconductivity measurements were made using a low-noise current amplifier and a Fourier transform infrared spectrometer (FTIR). The glowbar source of the FTIR was directed to the quantum dot detector under normal incidence. The current amplifier measured the response of the detector and was additionally used to bias the detector. The amplified signal is fed back to the FTIR for the interferogram, which was transformed to reveal the spectral response. Measurements at $90 \mathrm{~K}$ reveal a photoconductivity response in the range of $10-21 \mu \mathrm{m}$ with a peak at $\sim 17 \mu \mathrm{m}$, and the limit of the spectrometer at $\sim 20-24 \mu \mathrm{m}$. The signal is observed at a bias of $V_{\text {bias }}=0.5 \mathrm{~V}$. At zero bias, no signal is observed, and at biases just above $0.5 \mathrm{~V}$, the signal becomes too noisy to detect photocurrent. The photoconductivity spectra are shown in Fig. 4, with signal averaging used to clear up the noisy response. This response may be compared to that without illumination, also shown in Fig. 4, where no such features are observed, and only a noisy inconsistent interferogram is present. We believe that the observed response is due to intersubband transitions in the quantum dots, agreeing with photoluminescence and photocurrent 
measurements. We expect a stronger response, and at higher temperature, with the optimization of the quantum dot growth and the device structure design. As indicated from the photoluminescence spectra, the quantum dots have a relatively broad quantum dot size distribution. Upon improvement of the quantum dot size distribution, the response should further increase and become narrower as a larger number of quantum dots have similar band structures and subband energy separation. Further control over quantum dot size should also provide a means of tuning the detection wavelength in the mid- and far infrared.

This work is supported by the National Science Foundation under Grant No. ECS-9628973 and the Army Research Office under Grant No. DAAG 55-97-1-0156.

${ }^{1}$ L. C. West and S. J. Eglash, Appl. Phys. Lett. 46, 1156 (1985).

${ }^{2}$ B. F. Levine, in III-V Quantum Wells and Superlattices, edited by P. Bhattacharya (INSPEC, London, 1996), p. 288.
${ }^{3}$ H. Benisty, C. M. Sotomayor-Torres, and C. Weisbuch, Phys. Rev. B 44, 10945 (1991).

${ }^{4}$ I. Vurgaftman, Y. Lam, and J. Singh, Phys. Rev. B 50, 14309 (1994).

${ }^{5}$ L. Goldstein, F. Glas, J. Y. Marzin, M. N. Charasse, and G. LeRoux, Appl. Phys. Lett. 47, 1099 (1985).

${ }^{6}$ P. R. Berger, K. Chang, P. Bhattacharya, J. Singh, and K. K. Bajaj, Appl. Phys. Lett. 53, 684 (1988).

${ }^{7}$ G. J. Whaley and P. I. Cohen, Appl. Phys. Lett. 57, 144 (1990).

${ }^{8}$ S. Guha, A. Madhukar, and K. C. Rajkumar, Appl. Phys. Lett. 57, 2110 (1990).

${ }^{9}$ D. Leonard, M. Krishnamurthy, C. M. Reaves, S. P. Denbaars, and P. M. Petroff, Appl. Phys. Lett. 63, 3202 (1993).

${ }^{10}$ D. Klotzkin, K. Kamath, and P. Bhattacharya, IEEE Photonics Technol. Lett. 9, 1301 (1997).

${ }^{11}$ H. Drexler, D. Leonard, W. Hansen, J. P. Kotthaus, and P. M. Petroff, Phys. Rev. Lett. 73, 2252 (1994).

${ }^{12}$ D. Pan, Y. P. Zeng, M. Y. Kong, J. Wu, Y. Q. Zhu, C. H. Zhang, J. M. Li, and C. Y. Wang, Electron. Lett. 32, 1726 (1996).

${ }^{13}$ J. Phillips, K. Kamath, X. Zhou, N. Chervela, and P. Bhattacharya, Appl. Phys. Lett. 71, 2081 (1997).

${ }^{14}$ K. W. Berryman, S. A. Lyon, and M. Segev, Appl. Phys. Lett. 70, 1861 (1997). 